\title{
Continuous-variable-entanglement dynamics in structured reservoirs
}

\author{
Ruggero Vasile, ${ }^{1, *}$ Stefano Olivares, ${ }^{2,3, \dagger}$ Matteo G. A. Paris, ${ }^{2,3,4, \$}$ and Sabrina Maniscalco ${ }^{1, \S}$ \\ ${ }^{1}$ Turku Center for Quantum Physics, Department of Physics and Astronomy, University of Turku, 20014 Turun Yliopisto, Finland \\ ${ }^{2}$ Dipartimento di Fisica, Università degli Studi di Milano, I-20133 Milano, Italy \\ ${ }^{3}$ CNISM UdR Milano Università, I-20133 Milano, Italy \\ ${ }^{4}$ ISI Foundation, I-10133 Torino, Italy \\ (Received 12 October 2009; published 10 December 2009)
}

\begin{abstract}
We address the evolution of entanglement in bimodal continuous variable quantum systems interacting with two independent structured reservoirs. We derive an analytic expression for the entanglement of formation without performing the Markov and the secular approximations and study in details the entanglement dynamics for various types of structured reservoirs and for different reservoir temperatures, assuming the two modes initially excited in a twin-beam state. Our analytic solution allows us to identify three dynamical regimes characterized by different behaviors of the entanglement: the entanglement sudden death, the non-Markovian revival and the non-secular revival regimes. Remarkably, we find that, contrarily to the Markovian case, the short-time system-reservoir correlations in some cases destroy quickly the initial entanglement even at zero temperature.
\end{abstract}

DOI: 10.1103/PhysRevA.80.062324

PACS number(s): 03.67.Mn, 03.65.Yz

\section{INTRODUCTION}

Entanglement is an essential resource for quantum computation and communication protocols [1]. However, this fundamental quantum property is also fragile: the unavoidable interaction of quantum systems with their external environment leads to the irreversible loss of both quantum coherence (decoherence) and quantum correlations in multipartite systems [2-4].

A crucial requirement for a physical system to be of interest for quantum technologies is that the survival time of entanglement is longer than the time needed for information processing. Therefore it is important to develop a deep and precise understanding not only of the mechanisms leading to decoherence and entanglement losses but also of the dynamical features of these phenomena. Moreover, in view of recent developments in reservoir engineering techniques [5,6], it is interesting to investigate situations in which decoherence and disentanglement can be controlled, for example through a precise and accurate tuning of system and environment parameters.

In this paper, we consider the entanglement dynamics in noisy continuous variable (CV) quantum systems [7]. More specifically we focus our attention on a system of two noninteracting quantum harmonic oscillators bilinearly coupled to two independent structured reservoirs at temperature $T$. Our aim is to study the time evolution of the entanglement between the two oscillators for different temperature regimes, different system-reservoir parameters and different reservoir spectra. Rather than limiting ourselves to present a plethora of dynamical behaviors, we will try to identify general features in order to single out universal properties of the

\footnotetext{
*ruggero.vasile@utu.fi

†stefano.olivares@mi.infn.it

${ }^{\ddagger}$ matteo.paris@fisica.unimi.it

§sabrina.maniscalco@utu.fi
}

disentanglement process, namely, those properties that do not depend either on the specific model of reservoir chosen or on the specific initial value of the entanglement. Moreover, we also compare the differences in the dynamics arising from different spectral distributions of the reservoir in order to identify those physical contexts leading to stronger or weaker entanglement losses.

During the last decade numerous works dealing with losses and decoherence in bimodal CV quantum systems have appeared in the literature. In order to describe analytically the dynamics of such an open quantum system, approximations such as the Born-Markov and the secular (or rotating wave) approximations are typically performed [8-13]. The Markovian approximation basically consists in neglecting the short-time correlations between system and reservoir arising because of the structure of the reservoir spectrum. This approximation is often performed together with the weak system-reservoir coupling assumption, also known as Born approximation. The Born and the Markov approximations are generally related. Indeed, every time the coupling between the system and the environment is strong, and, therefore, the Born approximation is not appropriate, also the Markovian approximation cannot be consistently used. However, there exist situations of weak systemreservoir coupling and structured environment, where the system-reservoir correlations persist long enough to require a non-Markovian treatment, even in the weak coupling limit. In this paper we focus on these cases.

We also investigate the validity of the secular approximation (i.e., neglecting the counter-rotating terms in the Hamiltonian) and we find that, even for weak couplings, a correct description of the short-time dynamics must take into account the nonsecular terms. In more detail, the validity of the secular approximation sensibly depends both on the reservoir temperature and on the system-reservoir parameters.

Non-Markovian studies of bimodal CV quantum systems in a common reservoir have shown the existence of three different dynamical phases of the entanglement in the long time limit, namely, the sudden death, sudden death and re- 
vivals, and no-sudden death phases [14,15]. These phases depend not only on the system-reservoir parameters but also on the properties of the spectrum. In this paper we consider the case of two independent reservoirs and find a similar division in dynamical phases or regimes, namely, the entanglement sudden death (ESD), the non-Markovian revival (NMRev) and the nonsecular revival (NSRev) regimes. In our system, however, the no-sudden death phase appears only at zero temperature and under very specific conditions. Moreover, we have discovered that the revivals may be due to two different physical mechanisms, the non-Markovian finite reservoir memory or the presence of the non secular terms.

Recent literature on non-Markovian CV dynamics, in the common reservoir scenario, includes $[16,17]$, while the independent reservoirs case was considered using a phenomenological approach in [18] and using a numerical approach in $[19,20]$. In this paper, we extend in several directions the results we have obtained in [21], where we limited our study to the high- $T$ Ohmic reservoir in the secular approximation. Here, we solve the master equation for our system without performing the secular approximation and investigate quantitatively the entanglement dynamics using an analytic expression for the evolution of the entanglement of formation (EOF) [22-24]. We assume the two oscillators initially excited in a twin-beam state (TWB, sometimes also referred to as two-mode squeezed states) and consider Ohmic, subOhmic, and super-Ohmic reservoirs at any temperature.

The paper is organized as follows. In Sec. II, we introduce the physical system, the master equation and its general solution through the characteristic function approach. In Sec. III, we review some preliminary concepts about two-mode Gaussian states and we present the general solution of the master equation with an initial Gaussian state. We also present the TWB states, the concept of EOF for two-mode CV Gaussian states, and the types of reservoir spectra considered in the paper. In Sec. IV, we present a detailed investigation on the validity of the secular approximation in our model. In Sec. V we discuss the dynamics of entanglement and analyze the three emerging dynamical regimes: ESD, NMRev, and NSRev. Moreover, we give specific examples of the dynamics of the EOF focusing on the high- $T$ and low- $T$ regimes. Finally, in Sec. VI, we discuss and summarize our results, presenting conclusions and future prospectives.

\section{MASTER EQUATION}

We consider a system of two identical noninteracting quantum harmonic oscillators, each of them coupled to its own Bosonic structured reservoir. The total Hamiltonian can be written as

$$
\begin{aligned}
H= & \sum_{j=1,2} \hbar \omega_{0} a_{j}^{\dagger} a_{j}+\sum_{j=1,2} \sum_{k} \hbar \omega_{j k} b_{j k}^{\dagger} b_{j k}+\sum_{j=1,2} \sum_{k} \gamma_{j k}\left(a_{j}+a_{j}^{\dagger}\right) \\
& \times\left(b_{j k}+b_{j k}^{\dagger}\right),
\end{aligned}
$$

with $\omega_{0}$ as the oscillators frequency, $\omega_{1 k}$ and $\omega_{2 k}$ as the frequencies of the reservoirs modes, $a_{j}\left(a_{j}^{\dagger}\right)$ and $b_{j k}\left(b_{j k}^{\dagger}\right)$ as the annihilation (creation) operators of the system and reservoirs harmonic oscillators, respectively, and $\gamma_{j k}$ as the coupling between the $j$ th oscillator and the $k$ th mode of its environment. In the following, we assume that the reservoirs have the same spectrum and are equally coupled to the oscillators.

Since we are interested in the dynamics of the two oscillators only, we adopt a density matrix approach through the following local in time master equation [25],

$$
\begin{aligned}
\dot{\rho}(t)= & \sum_{j} \frac{1}{i \hbar}\left[H_{j}^{0}, \rho(t)\right]-\Delta(t)\left\{X_{j},\left[X_{j}, \rho(t)\right]\right\}+\Pi(t) \\
& \times\left\{X_{j},\left[P_{j}, \rho(t)\right]\right\}+\frac{i}{2} r(t)\left[X_{j}^{2}, \rho(t)\right]+-i \gamma(t) \\
& \times\left\{X_{j},\left[P_{j}, \rho(t)\right]\right\},
\end{aligned}
$$

where $\rho(t)$ is the reduced density matrix, $H_{j}^{0}$ is the free Hamiltonian of the $j$ th oscillator, and $X_{j}=\left(a_{j}+a_{j}^{\dagger}\right) / \sqrt{2}$ and $P_{j}=i\left(a_{j}^{\dagger}-a_{j}\right) / \sqrt{2}$ are the quadrature operators. The effect of the interaction with the reservoirs is contained in the timedependent coefficients of Eq. (2). The quantities $\Delta(t)$ and $\Pi(t)$ describe diffusion processes, $\gamma(t)$ is a damping term and $r(t)$ renormalizes the free oscillator frequency $\omega_{0}$.

It is worth noting that the master equation (2) is exact since neither the Born-Markov approximation nor the secular approximation have been performed. The time dependent coefficients can be expressed as power series in the systemreservoir coupling constant. For weak couplings one can stop the expansion to second order and obtain analytic solutions for the coefficients. In the case of reservoirs in thermal equilibrium at temperature $T$, characterized by a spectral density $J(\omega)$, these expressions read

$$
\begin{gathered}
\Delta(t)=\alpha^{2} \int_{0}^{t} d s \int_{0}^{+\infty} d \omega J(\omega)[2 N(\omega)+1] \cos (\omega s) \cos \left(\omega_{0} s\right), \\
\Pi(t)=\alpha^{2} \int_{0}^{t} d s \int_{0}^{+\infty} d \omega J(\omega)[2 N(\omega)+1] \cos (\omega s) \sin \left(\omega_{0} s\right), \\
\gamma(t)=\alpha^{2} \int_{0}^{t} d s \int_{0}^{+\infty} d \omega J(\omega) \sin (\omega s) \sin \left(\omega_{0} s\right) \\
r(t)=\alpha^{2} \int_{0}^{t} d s \int_{0}^{+\infty} d \omega J(\omega) \sin (\omega s) \cos \left(\omega_{0} s\right)
\end{gathered}
$$

where $N(\omega)=\left[\exp \left(\hbar \omega / k_{B} T\right)-1\right]^{-1}$ is the mean number of photons with frequency $\omega$, and $\alpha$ is the dimensionless system-reservoir coupling constant.

By using the characteristic function approach [26], the solution of the master equation (2) may be written as

$$
\chi_{t}(\Lambda)=e^{-\Lambda^{T}[\bar{W}(t) \oplus \bar{W}(t)] \Lambda} \chi_{0}\left(e^{-\Gamma(t) / 2}\left[R^{-1}(t) \oplus R^{-1}(t)\right] \Lambda\right),
$$

where $\chi_{t}(\Lambda)$ is the characteristic function at time $t, \chi_{0}$ is the characteristic function at the initial time $t=0, \quad \Lambda$ 
$=\left(x_{1}, p_{1}, x_{2}, p_{2}\right)$ is the two-dimensional phase space variables vector, $\Gamma(t)=2 \int_{0}^{t} \gamma\left(t^{\prime}\right) d t^{\prime}$, and $\bar{W}(t)$ and $R^{-1}(t)$ are the $2 \times 2$ matrices. The former matrix is given by

$$
\bar{W}(t)=e^{-\Gamma(t)}\left[R^{-1}(t)\right]^{T} W(t) R^{-1}(t),
$$

while the latter one, $R(t)$, contains rapidly oscillating terms. In the weak coupling limit $R(t)$ takes the form

$$
R(t)=\left(\begin{array}{cc}
\cos \omega_{0} t & \sin \omega_{0} t \\
-\sin \omega_{0} t & \cos \omega_{0} t
\end{array}\right) .
$$

Finally, $W(t)=\int_{0}^{t} e^{\Gamma(s)} \bar{M}(s) d s$ with $\bar{M}(s)=R^{T}(s) M(s) R(s)$ and

$$
M(s)=\left(\begin{array}{cc}
\Delta(s) & -\Pi(s) / 2 \\
-\Pi(s) / 2 & 0
\end{array}\right) .
$$

The coefficient $r(t)$ does not appear explicitly in the characteristic function solution because its contribution is negligible in the weak coupling regime [26]. The characteristic function approach of [26] is equivalent to other methods of solution of the master equation (2), as the Feynman-Vernon influence functional technique [27]. In this paper, we use the former one because it allows us to obtain an analytic solution in the weak coupling limit.

\section{ENTANGLEMENT DYNAMICS FOR GAUSSIAN STATES}

In this section, we derive the explicit analytic solution for the characteristic function in the weak coupling limit already obtained in [26]. Remarkably, the evolution induced by the master equation (2) corresponds to a Gaussian map, i.e., an initial Gaussian state maintains its character. It is thus possible to obtain the expression of the covariance matrix at time $t$ and then evaluate the EOF at any time for the two modes initially excited in a TWB state. We also present the classes of spectral densities considered in the paper and show how the form of the time-dependent coefficients ruling the dynamics can be simplified in the non-Markovian time scale.

\section{A. Analytic solution in the weak coupling limit}

Let us consider two-mode Gaussian states, i.e., those states characterized by a Gaussian characteristic function

$$
\chi_{0}(\Lambda)=\exp \left\{-\frac{1}{2} \Lambda^{T} \sigma_{0} \Lambda-i \Lambda^{T} \overline{\mathbf{X}}_{i n}\right\}
$$

We indicate with $\sigma_{0}$ the initial covariance matrix

$$
\sigma_{0}=\left(\begin{array}{ll}
\mathbf{A}_{\mathbf{0}} & \mathbf{C}_{\mathbf{0}} \\
\mathbf{C}_{\mathbf{0}}^{\mathbf{T}} & \mathbf{B}_{\mathbf{0}}
\end{array}\right)
$$

where $\mathbf{A}_{\mathbf{0}}=a \rrbracket, \mathbf{B}_{\mathbf{0}}=b 1$, and $\mathbf{C}_{\mathbf{0}}=\operatorname{Diag}\left(c_{1}, c_{2}\right)$, with $a, b>0$ and $c_{1}, c_{2}$ real numbers, and $\mathbb{1}$ the $2 \times 2$ identity matrix. Moreover,

$$
\overline{\mathbf{X}}_{\text {in }}=\operatorname{Tr}\left[\rho(0)\left(X_{1}, P_{1}, X_{2}, P_{2}\right)^{T}\right] .
$$

If $c_{1}=c_{2}=0$ the initial covariance matrix is block diagonal and the corresponding state is separable. Since each oscilla- tor only interacts with its own environment, an initial separable state remains separable during all the evolution. For initial entangled states, however, the entanglement dynamics will in general depend on the initial value of the entanglement and on reservoir properties such as the spectral distribution, the temperature and the coupling constants.

Since the evolution maintains the Gaussian character the evolved state is a two-mode Gaussian state with mean and covariance matrix given by

$$
\begin{gathered}
\overline{\mathbf{X}}_{t}=e^{-\Gamma(t) / 2}(R \oplus R) \overline{\mathbf{X}}_{i n}, \\
\sigma_{t}=e^{-\Gamma(t)}(R \oplus R) \sigma_{0}(R \oplus R)^{T}+2\left(\bar{W}_{t} \oplus \bar{W}_{t}\right),
\end{gathered}
$$

Using Eqs. (4)-(7) we obtain

$$
\begin{aligned}
\bar{W}_{t}= & e^{-\Gamma(t)} \int_{0}^{t} e^{\Gamma(s)}\left[\frac{\Delta(s)}{2} 1+\frac{\Delta(s)}{2} \mathbf{C}_{2}(t-s)\right. \\
& \left.-\frac{\Pi(s)}{2} \mathbf{S}_{2}(t-s)\right] d s,
\end{aligned}
$$

where

$$
\begin{aligned}
\mathbf{C}_{2}(t) & =\left(\begin{array}{cc}
\cos 2 \omega_{0} t & -\sin 2 \omega_{0} t \\
-\sin 2 \omega_{0} t & -\cos 2 \omega_{0} t
\end{array}\right), \\
\mathbf{S}_{2}(t) & =\left(\begin{array}{cc}
\sin 2 \omega_{0} t & \cos 2 \omega_{0} t \\
\cos 2 \omega_{0} t & -\sin 2 \omega_{0} t
\end{array}\right) .
\end{aligned}
$$

The covariance matrix at time $t$ is given by

$$
\boldsymbol{\sigma}_{t}=\left(\begin{array}{c|c}
\boldsymbol{A}_{t} & \boldsymbol{C}_{t} \\
\hline \boldsymbol{C}_{t}^{T} & \boldsymbol{A}_{t}
\end{array}\right)
$$

with

$$
\boldsymbol{A}_{t}=\boldsymbol{A}_{0} e^{-\Gamma}+\left[\begin{array}{cc}
\Delta_{\Gamma}+\left(\Delta_{\mathrm{co}}-\Pi_{\mathrm{si}}\right) & -\left(\Delta_{\mathrm{si}}-\Pi_{\mathrm{co}}\right) \\
-\left(\Delta_{\mathrm{si}}-\Pi_{\mathrm{co}}\right) & \Delta_{\Gamma}-\left(\Delta_{\mathrm{co}}-\Pi_{\mathrm{si}}\right)
\end{array}\right],
$$

and

$$
\boldsymbol{C}_{t}=\left[\begin{array}{cc}
c e^{-\Gamma} \cos \left(2 \omega_{0} t\right) & c e^{-\Gamma} \sin \left(2 \omega_{0} t\right) \\
c e^{-\Gamma} \sin \left(2 \omega_{0} t\right) & -c e^{-\Gamma} \cos \left(2 \omega_{0} t\right)
\end{array}\right],
$$

where we have introduced the function

$$
\Delta_{\Gamma}(t)=e^{-\Gamma(t)} \int_{0}^{t} e^{\Gamma(s)} \Delta(s) d s
$$

and the secular coefficients

$$
\begin{aligned}
& \Delta_{\mathrm{co}}(t)=e^{-\Gamma(t)} \int_{0}^{t} e^{\Gamma(s)} \Delta(s) \cos \left[2 \omega_{0}(t-s)\right] d s, \\
& \Delta_{\mathrm{si}}(t)=e^{-\Gamma(t)} \int_{0}^{t} e^{\Gamma(s)} \Delta(s) \sin \left[2 \omega_{0}(t-s)\right] d s, \\
& \Pi_{\mathrm{co}}(t)=e^{-\Gamma(t)} \int_{0}^{t} e^{\Gamma(s)} \Pi(s) \cos \left[2 \omega_{0}(t-s)\right] d s,
\end{aligned}
$$




$$
\Pi_{\mathrm{si}}(t)=e^{-\Gamma(t)} \int_{0}^{t} e^{\Gamma(s)} \Pi(s) \sin \left[2 \omega_{0}(t-s)\right] d s .
$$

The explicit analytic expression of the coefficients above depends on both the reservoir spectral density and the temperature.

To further simplify the solution it is common to perform the so-called secular approximation. This approximation amounts at neglecting rapidly oscillating terms in the solution of the master equation. In our case this means to assume that the coefficients (20) average out to zero. Stated another way, the secular solution is a coarse-grained expression of the exact one. In the next section we will critically examine the validity of the secular approximation and derive the conditions of validity for different reservoir spectra and systemreservoir parameters.

From now on we focus on TWB states, i.e., a set of Gaussian states whose covariance matrix (9) has $a=b$ $=\cosh (2 r) / 2$ and $c_{1}=-c_{2}=\sinh (2 r) / 2$, with $r>0$ the squeezing parameter. Being pure states, their amount of entanglement is given by the entropy of entanglement $E_{0}(r)$ $=2\left[\cosh ^{2} r \ln (\cosh r)-\sinh ^{2} r \ln (\sinh r)\right]$ and, hence, it increases for increasing values of $r$.

\section{B. Entanglement of Formation}

A convenient and useful way of looking at the entanglement evolution in $\mathrm{CV}$ systems is by means of the EOF $[22,23]$. This quantity corresponds to the minimal amount of entanglement of any ensemble of pure bipartite states realizing the given state. In general it is not a simple task to derive an expression of the EOF for arbitrary states. Recently, its expression for an arbitrary bimodal Gaussian state has been obtained in [24].

We assume here that the initial state is a symmetric bipartite Gaussian state with covariance matrix given by Eq. (9). As we mentioned above, when this state interacts with two identical independent reservoirs, the Gaussian character is preserved and the evolved covariance matrix is given by Eq. (16). Due to the symmetry of the evolved state, the EOF is given by [23]

$$
E_{F}=\left(x_{m}+\frac{1}{2}\right) \ln \left(x_{m}+\frac{1}{2}\right)-\left(x_{m}-\frac{1}{2}\right) \ln \left(x_{m}-\frac{1}{2}\right),
$$

with $x_{m}=\left(\widetilde{\kappa}_{-}^{2}+1 / 4\right) /\left(2 \widetilde{\kappa}_{-}\right), \widetilde{\kappa}_{-}=\sqrt{\left(a_{n}-c_{+}\right)\left(a_{n}-c_{-}\right)}$being the minimum symplectic eigenvalue of the CM $\boldsymbol{\sigma}_{t}$, and

$$
\begin{gathered}
a_{n}=\sqrt{I_{1}}, \\
c_{ \pm}=\sqrt{\frac{I_{1}^{2}+I_{3}^{2}-I_{4} \pm \sqrt{\left(I_{1}^{2}+I_{3}^{2}-I_{4}\right)^{2}-\left(2 I_{1} I_{3}\right)^{2}}}{2 I_{1}}},
\end{gathered}
$$

where $I_{1}=\operatorname{det}\left[\boldsymbol{A}_{t}\right], I_{3}=\operatorname{det}\left[\boldsymbol{C}_{t}\right]$ and $I_{4}=\operatorname{det}\left[\boldsymbol{\sigma}_{t}\right]$ are the symplectic invariants of $\boldsymbol{\sigma}_{t}$. Inserting Eqs. (16)-(19) and (20a)(20d) into Eqs. (21)-(23) one obtains the analytic expression of the EOF for our system.

\section{Modeling the reservoir}

In order to obtain explicit expressions for the EOF, we need to specify the properties of the Bosonic reservoirs. We consider environments in thermal equilibrium at temperature $T$ and we focus on the following class of Ohmic-like spectral distributions with an exponential cutoff function

$$
J_{s}(\omega)=\omega_{c}\left(\frac{\omega}{\omega_{c}}\right)^{s} e^{-\omega / \omega_{c}},
$$

where $\omega_{c}$ is the cut-off frequency. The case $s=1$ corresponds to an Ohmic reservoir spectrum, characterized by a linear dependence on the frequency for $\omega \ll \omega_{c}$. For $s>1$ the spectrum is known as super-Ohmic while $s<1$ describes a subOhmic spectral distribution. For the sake of concreteness, in the following we consider the $s=3$ super-Ohmic and the $s$ $=1 / 2$ sub-Ohmic cases. A more detailed discussion about the properties of these spectral distributions can be found, e.g., in [28].

A closed form for the expressions of the time-dependent coefficients given in Eq. (3) can be obtained in the high- $T$ and zero- $T$ limits, i.e., for $2 N(\omega)+1 \approx \frac{2 k_{B} T}{\hbar \omega}$ and $2 N(\omega)+1$ $\approx 1$, respectively (see Appendix). Therefore, we focus on these two regimes. Inserting now the spectral distributions of Eq. (24), with $s=1, s=1 / 2$, and $s=3$, into Eq. (3) allows to determine the analytic form of the time-dependent coefficients. We notice that, in all three cases, after a time $t \approx \tau_{c}$ $=\omega_{c}^{-1}$, the coefficients attain their Markovian stationary values and the system behaves according to the predictions of the Markovian theory. Here, we are particularly interested in the non-Markovian short-time dynamics, and therefore we will focus on times $t \leq \tau_{c}$. In this time interval, and in the weak coupling limit, we can expand the exponential terms appearing in Eqs. (19) and (20) in Taylor series. For example Eq. (19) becomes

$\Delta_{\Gamma}(t) \simeq \int_{0}^{t} \Delta(s) d s-\Gamma(t) \int_{0}^{t} \Delta(s) d s+\int_{0}^{t} \Gamma(s) \Delta(s) d s+O\left(\alpha^{4}\right)$.

Since $\Delta(t) \propto \alpha^{2}$ and $\Gamma(t) \propto \alpha^{2}$, in the weak coupling limit $(\alpha \ll 1)$ and for short non-Markovian times the first term dominates, and hence, it is the only one that will be retained.

\section{ON THE VALIDITY OF THE SECULAR APPROXIMATION}

In this section, we question the validity of the secular approximation by comparing the entanglement dynamics with or without the secular terms (20). As we will see, in general, the secular terms do influence the behavior of the entanglement in the short non-Markovian time scale. Depending on the value of certain parameters, however, the secular approximation in some cases turns out to give a good description of the dynamics. We have identified as main parameters influencing the time evolution the reservoir temperature, the parameter $x=\omega_{c} / \omega_{0}$, and the form of the reservoir spectrum. We will discuss the effect of these parameters separately in the following three subsections dealing with the dynamics for high- $T$ reservoirs, $T=0$ reservoir, and with a comparison between different reservoir spectra. We will also consider if and how the validity of the secular approximation 


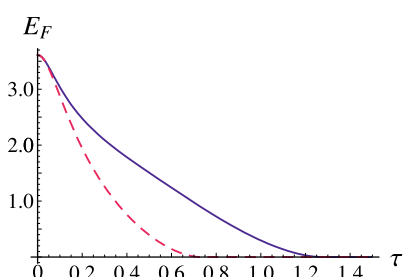

(a)

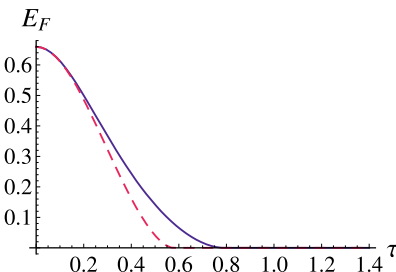

(b)

FIG. 1. (Color online) Comparison between the exact EOF dynamics (solid blue line) and the secular approximate dynamics (dashed red line) as a function of $\tau=\omega_{c} t$, with $x=10$, (a) $r=2$, and (b) $r=0.5$. We set $k_{B} T / \hbar \omega_{c}=100$ and $\alpha=0.1$.

depends on the initial state of the system and, in particular, on the initial squeezing parameter $r$ of TWBs.

We recall that in this paper, we will focus only on the dynamics of entanglement. Different observables of the system may show different sensitivity to the secular approximation. Indeed, we know that there exist a class of observables, e.g., the energy of the system oscillators, that are not influenced at all by this approximation [26].

\section{A. High-temperatures regime}

We begin addressing the high-temperature limit $k_{B} T$ $\gg \hbar \omega_{0}, \hbar \omega_{c}$, i.e., when the classical thermal energy $k_{B} T$ is much larger than the typical energies exchanged in our system. In the following analysis we choose a temperature such that $k_{B} T / \hbar \omega_{c}=100$, thus, we can examine scenarios, in which $x=\omega_{c} / \omega_{0} \geq 0.1$.

We start analyzing the differences in the EOF evolution between the secular result and the exact one in the case of an Ohmic distribution and $x=10$. In Fig. 1 we plot the time evolution of the EOF calculated using the secular approximated solution and using the exact solution in the regime $x$ $\gg 1$ for two different initial TWB states. For both initial conditions the secular approximation fails. Remarkably, the exact solution containing the nonsecular terms predicts a much longer disentanglement time. Furthermore the difference in the disentanglement time predicted by the exact and secular results increases for increasing values of initial entanglement, i.e., for larger values of $r$. This result is qualitatively independent of the analytic form of the reservoir spectrum, as we will see in Sec. IV C were the effect of different spectra is considered.

For intermediate values of the parameter $x, x \leq 1$, we observe a stronger dependence on the initial value of entanglement. In Fig. 2, indeed, we see that for $x=0.2$ and $r=0.1$ (small initial entanglement) the secular approximation works well, but for higher values of the initial entanglement, $r=1$, nonsecular oscillations, absent in the secular approximated solution, are clearly visible in the exact dynamics.

For $x \ll 1$, finally, the nonsecular oscillations decrease in amplitude as the effective coupling with the environment decreases and the secular coarse-grained solution describes well the dynamics of the entanglement in the short nonMarkovian time-scale, independently from the initial condition. This behavior is in agreement with the results of [29] where the weak coupling limit of the master equation for

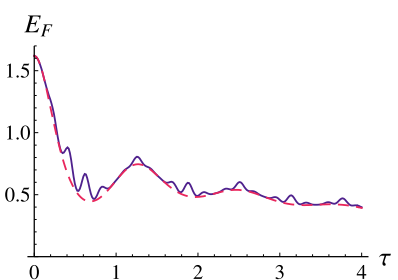

(a)

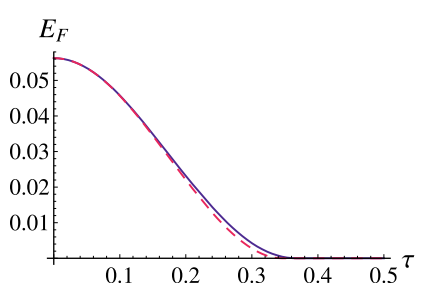

(b)
FIG. 2. (Color online) Comparison between the exact EOF dynamics (solid blue line) and the secular approximate dynamics (dashed red line) as a function of $\tau=\omega_{c} t$, with $x=0.2$, (a) $r=1$, and (b) $r=0.1$. We set $k_{B} T / \hbar \omega_{c}=100$ and $\alpha=0.1$.

quantum Brownian motion is discussed. In particular, in [29], it is shown that, in the high-temperature and weak coupling limits, the secular approximated master equation is accurate only in the regime $x \ll 1$, while for the other regimes the system behaves as if it were subjected to a squeezed reservoir.

Summarizing, for high- $T$ Ohmic reservoirs, the secular approximation holds only in the regime $x \ll 1$. This result is also valid for the sub-Ohmic and super-Ohmic environments.

\section{B. Zero-temperature regime}

From previous studies on open quantum systems interacting with zero-temperature reservoirs we expect on the one hand a slower loss of entanglement [8] and on the other hand more pronounced non-Markovian features [30], with respect to the $T \neq 0$ case. We will have a closer look at these general features of the dynamics in Sec. $\mathrm{V}$ and focus here on the validity of the secular approximation.

We consider as an example a super-Ohmic reservoir with $x=0.3$ and look at the dynamics of a TWB with a small amount of initial entanglement, $r=0.01$. As shown in Fig. 3, the exact and the secular approximated dynamics sensibly agree in this situation. We have carefully examined the dynamical behavior for other values of $x$ and of the initial squeezing parameter $r$ reaching the conclusion that this is quite a general property of the system. Therefore, in the description of bimodal CV quantum systems interacting with zero- $T$ reservoirs, the secular approximation can always be preformed and the effect of the nonsecular terms is always negligible. This is a consequence of the fact that the secular

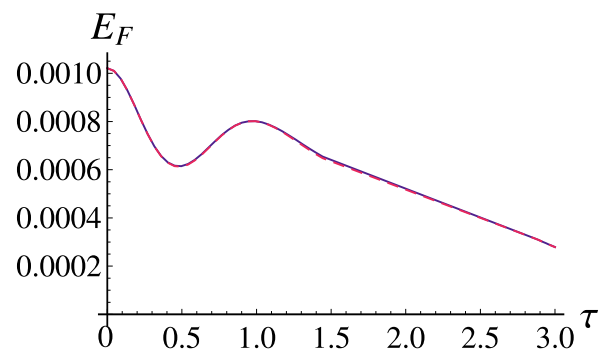

FIG. 3. (Color online) The exact (blue solid line) and the secular approximated dynamics (red dashed line) of the $E_{F}$ vs $\tau=\omega_{c} t$ in a super-Ohmic reservoir at zero temperature for $\alpha=0.1, r=0.01$, and $x=0.3$. The two curves almost overlap perfectly. 


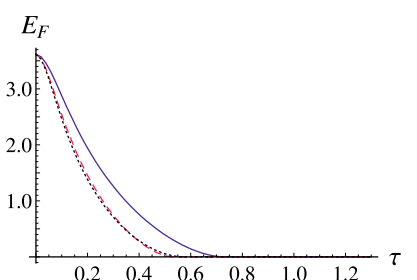

(a)

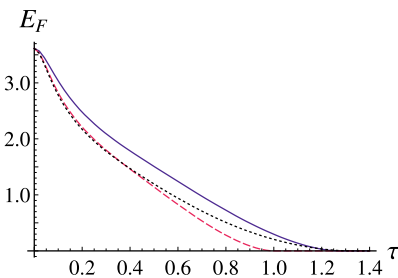

(b)

FIG. 4. (Color online) Comparison between the dynamics of $E_{F}$ for the Ohmic (blue solid line), sub-Ohmic (red dashed line), and super-Ohmic (black dotted line) reservoir spectra using (a) the secular approximated solution and (b) the exact solution in the hightemperature limit with $k_{B} T / \hbar \omega_{c}=100, \alpha=0.1, r=2$, and $x=10$.

terms (20) are temperature dependent through the diffusion coefficients $\Delta(t)$ and $\Pi(t)$, and at $T=0$ their contribution is rather small.

\section{Dependency on the reservoir spectrum}

To conclude our analysis of the secular approximation we look at the discrepancy between the secular and exact solutions for Ohmic, sub-Ohmic and super Ohmic reservoirs. Since in the zero- $T$ case the secular approximation always works well, we focus on the high- $T$ case and in particular on the $x \gg 1$ regime, where the differences in the dynamics of the EOF are most pronounced.

In Fig. 4 we compare the dynamics of $E_{F}$ for the Ohmic, sub-Ohmic, and super-Ohmic reservoirs as given by the secular approximation, Fig. 4(a), with the exact case, Fig. 4(b), for $x=10$ and $r=2$. Comparing the two figures one clearly sees that the secular approximation does not affect the dynamics in an equal way for the three different spectral distributions. The exact disentanglement time is almost tripled for the super-Ohmic environment and doubled for the Ohmic case. The sub-Ohmic case is less affected. In all cases, however, and for all values of initial entanglement, the exact calculation predicts a longer survival time of entanglement.

\section{ENTANGLEMENT DYNAMICS}

\section{A. General features: Three different dynamical regimes}

In discrete variable quantum systems the phenomenon of ESD has recently received a lot of attention [31]. In that context, the basic system studied consists of two qubits interacting with either independent or common reservoirs. An exact solution has been derived both for independent [32] and for common reservoirs [33], and it has been shown that revivals of entanglement due to the reservoir memory may occur after an initial sudden death interval. The common reservoir scenario is generally characterized by a nonzero long-time entanglement due to both the reservoir-mediated interaction between the qubits and the existence of a decoherence free subradiant state acting as an entanglement trap [34-36]. In the independent reservoir case, on the contrary, in the long time limit one always observes complete disentanglement.
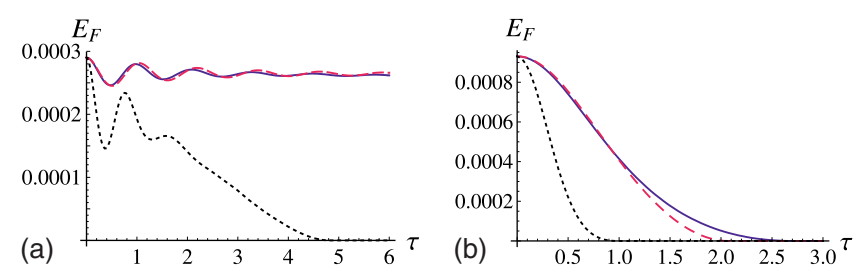

FIG. 5. (Color online) Dynamics of $E_{F}$ at zero temperature for $\alpha=0.1$ in the case of Ohmic (blue line), sub-Ohmic (red line), and super-Ohmic (black line) reservoirs for (a) $x=0.2$ and $r=0.005$ and for (b) $x=10$ and $r=0.01$.

In the following, we focus on the case in which the carriers of quantum information are continuous, instead of discrete, variable quantum systems. While the theoretical treatments of CV quantum channels are fundamentally different from the one of discrete channels, we find that some similarities in the entanglement dynamics do exist. In particular, in the common reservoir scenario, due to the environmentmediated interaction between the two $\mathrm{CV}$ channels, the asymptotic long-time entanglement maybe nonzero, even for high- $T$ reservoirs $[14,15]$. Moreover, non-Markovian studies show the occurrence of revivals of entanglement both in the common and in the independent reservoir cases $[14,15,21]$.

Here we show that, for independent reservoirs and for $x$ $\gg 1$, the phenomenon of ESD occurs both in the high- $T$ and, for $r \ll 1$, in the zero- $T$ cases, independently from the reservoirs spectra, as one can see from Figs. 1, 4, and 5(b). The ESD regime can directly be linked to the behavior of the time-dependent coefficients appearing in the master equation (2). For high- $T$ and $x \gg 1$, indeed, independently from the reservoir spectra, the time dependent coefficients are always positive at every time instant [28]. In systems described by time-convolutionless master equations non-Markovian features typically occur when the time-dependent coefficients temporarily attain negative values $[37,38]$. When this happens revivals of entanglement may occur since the system restores partially the quantum coherence previously lost due to the interaction with the environment. An example of nonMarkovian revivals due to the reservoir memory effects, and therefore connected to the negativity of the time dependent coefficients, is shown in Fig. 6, where we plot the EOF dynamics for an Ohmic reservoir in the high-T limit. In general non-Markovian revivals of entanglement occur for $x \ll 1$. In

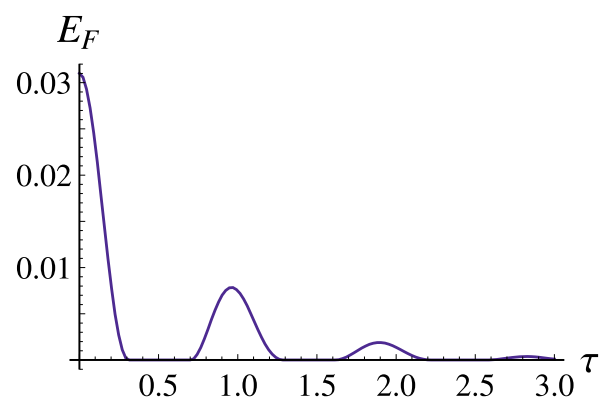

FIG. 6. (Color online) $E_{F}$ dynamics vs $\tau=\omega_{c} t$ for an high- $T$ Ohmic reservoir with $k_{B} T / \hbar \omega_{c}=100, \alpha=0.1, x=0.15$, and $r=0.06$. Note the presence of entanglement revivals due to negative values of the master equation coefficients. 


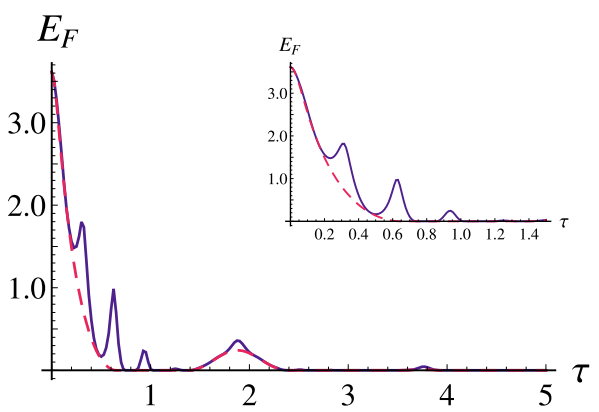

FIG. 7. (Color online) $E_{F}$ for a Sub-Ohmic reservoir in the high-T limit $\left(k_{B} T / \hbar \omega_{c}=100\right)$ with $\alpha=0.1, r=2$, and $x=0.3$. The solid blue line is the exact solution while the dashed red line is the secular solution. The inset is a magnification of the temporal region $0 \leq \tau \leq 1.5$.

this case we have seen that the secular approximation works well and we know from previous studies (See [28,39]) that the time dependent coefficients attain negative values for all reservoir spectra.

Our exact approach allows us, moreover, to single out another type of revivals, not related directly to the negativity of the time-dependent coefficients, and therefore, on the reservoir memory, but rather on the presence of nonsecular terms. An example of the nonsecular revivals is given in Fig. 7, where the exact and the secular solutions are compared. In this case the time-dependent coefficients are positive for $\tau$ $\lesssim 1.4$ and the partial and temporary restoration of entanglement in that time interval is due to the presence of the counter-rotating terms in the microscopic Hamiltonian model. It is often believed that the nonsecular or counterrotating terms significantly affect the dynamics only in the strong coupling limit. For discrete variable systems indeed, a very recent study has shown the non-negligible effect of nonsecular terms in the strong coupling limit [40]. In this case, the authors show that the exact dynamics causes a faster loss of entanglement with respect to the secular case. Here we show that also in the weak coupling limit these terms give a non-negligible contribution in the short non-Markovian time scale. In general, for intermediate values of $x$, the dynamics will display both nonsecular and non-Markovian revivals. Indeed, if we look at the dynamics for $\tau \gtrsim 1.4$ we see that a non-Markovian revival, with a superimposed nonsecular revival, occurs at $1.5 \leqq \tau \lesssim 2.5$, as shown in Fig. 7(b). The border between the NMRev and the NSRev dynamical regimes is therefore a blurred region in which both effects occur at the same time. In this case both the reservoir memory and the nonsecular terms contribute to the reappearance of previously lost entanglement.

A more detailed discussion is required to explain the presence of ESD in the zero-temperature cases shown in Figs. 5(a) and 5(b). The Markovian theory of two-mode continuous variable channels predicts, for both the common and the independent reservoirs, the existence of a finite time of disentanglement for an initial TWB state when $T>0$ [8]. In the independent reservoirs model at $T=0$, however, the Markovian disentanglement time is infinite (no ESD). Since the exact master equation (2) coincides with the approximate BornMarkov master equation for weak couplings and for times longer than the reservoirs correlation time, one would expect our non-Markovian model to give the same prediction for the disentanglement time than the Markovian one. Stated another way, one would not expect ESD.

However, one should keep in mind that the Markovian approximation is always a coarse graining in time and therefore it does not allow us to predict the short time nonMarkovian behavior. If at short times the initial entanglement is lost and no non-Markovian revivals occur, entanglement cannot reappear at longer times. Consequently the entanglement will remain zero also in the asymptotic Markovian long-time region. This is exactly what may happen when the initial amount of entanglement is small $(r \ll 1)$. In this case, indeed, for some reservoirs spectra and values of $x$, the short time non-Markovian dynamics shows the occurrence of sudden death of entanglement. Since the state remains separable for times greater than the reservoirs correlation time, entanglement revivals cannot appear.

For higher values of initial entanglement, on the other hand, the exact non-Markovian theory does not lead to a sudden death in the short time scale, thus the state is still entangled when reaching the Markovian time-region and therefore the Markovian prediction of an infinite disentanglement time at $T=0$ still holds.

Summarizing, for $x \gg 1$ ESD occurs, independently from the reservoir spectrum, both in high $T$ reservoirs (for all values of $r$ ) and in zero- $T$ reservoirs (for $r \ll 1$ ). When $x \ll 1$ we are generally in the NMRev region, independently from the reservoir spectrum. One should note, however, that if the initial entanglement is very small $(r \ll 0.1)$ entanglement oscillations do not have time to take place and only ESD is observed. For intermediate values of $x$ the dynamical regimes strongly depend both on the reservoir spectrum and on the initial entanglement. More specifically, for high- $T$ reservoirs one can have any of the three ESD, NMRev and NSRev behaviors, as well as a combination of NMRev and NSRev. For zero- $T$ reservoirs the ESD or NMRev regimes exist only if the initial amount of entanglement is small $(r \ll 1)$, for other initial values of $r$ entanglement is never lost, in accordance with the Markovian theory.

\section{B. Comparative study of Ohmic, sub-Ohmic, and super-Ohmic reservoirs}

In this section, we investigate the differences in the loss of entanglement due to different reservoir spectra. Different physical systems are characterized by different environmental spectral densities, e.g., it is well known that solid-state systems are subjected to sub-Ohmic $1 / f$ noise. Such a comparative study, hence, allows to understand which physical context is more "quantum information friendly," in the sense of allowing entanglement to live longer.

We begin considering the high- $T$ reservoir case. In Fig. 4(b), we have seen that in the ESD regime and for high temperatures the behavior of entanglement is qualitatively similar. The disentanglement time is not strongly dependent on the reservoir spectrum and the sub-Ohmic environment displays the faster loss of entanglement. For intermediate values of $x$, however, the time evolution of $E_{F}$ shows a much 


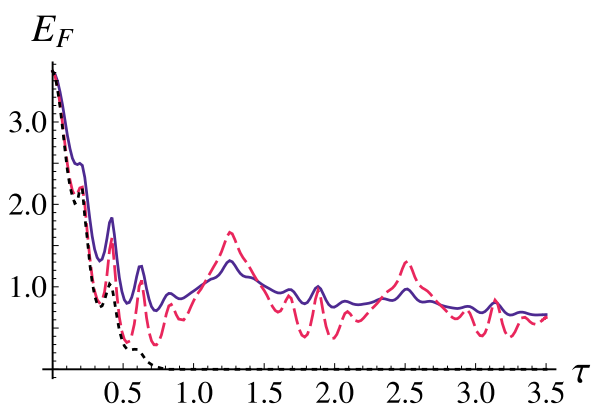

FIG. 8. (Color online) Dynamics of the $E_{F}$ in the high-T temperature limit $k_{B} T / \hbar \omega_{c}=100$ for $r=2$, and $x=0.2$ in the case of Ohmic (blue solid line), sub-Ohmic (red dashed line) and superOhmic (black dotted line) environments.

richer behavior and a much stronger dependence on the form of the spectrum, as one can see in Fig. 8. In this case the super-Ohmic environment shows a much faster loss of entanglement than the Ohmic and sub-Ohmic. In the Ohmic and sub-Ohmic cases one can clearly see the nonsecular oscillations superimposed to the non-Markovian oscillations in the dynamics of $E_{F}$, the latter ones having longer period and larger amplitude.

The entanglement dynamics for zero- $T$ reservoirs is plotted in Fig. 5 for (a) $x=0.2$ and (b) $x=10$. Also in this case the super-Ohmic reservoir shows a much faster entanglement loss than the sub-Ohmic and the Ohmic ones. This is especially evident in the intermediate $x$ region of Fig. 5(a). The Markovian and RW theory of entanglement dynamics for TWBs in independent reservoirs predicts that the disentanglement time (or separability time) should approach infinity for $T \rightarrow 0$ [8]. Our results demonstrate that this conclusion, in the case of small initial entanglement, is a consequence of the Markovian approximation and that the exact non-Markovian theory predicts that, even for weak system-reservoir coupling, in the $x \gg 1$ region the entanglement survives only for a short time. For intermediate values of $x$, however, the disentanglement time approaches the Markovian prediction for the sub-Ohmic and Ohmic cases. Indeed we see that after initial non-Markovian oscillations $E_{F}$ approaches its stationary nonzero Markovian value.

\section{SUMMARY AND CONCLUSIONS}

In this paper, we have studied a bimodal CV quantum system interacting with independent structured reservoirs in thermal equilibrium. We focus on the dynamics of the entanglement, as measured by the entanglement of formation, for the two modes initially excited in a twin-beam state and for different reservoir spectral distributions (Ohmic, subOhmic, and super-Ohmic). Under the only assumption of weak coupling we have obtained an exact analytic solution for the time-dependent two-mode covariance matrix describing the state of our system in the short time non-Markovian limit.

In the first part of the paper, we unravel the role of the secular approximation in our specific system and in particular in the context of the entanglement dynamics. By compar- ing the exact solution with the solution in the secular assumption we found that, in general, the high- $T$ dynamics is affected by this approximation, while the $T=0$ case is not. More specifically at high temperatures we have shown that for $x \gg 1$ both the exact and secular solutions predict the occurrence of ESD. However the entanglement persists longer in the exact solution. On the contrary in the limit of $x \ll 1$ the solutions agree. These two results are independent from the analytic expression of the reservoirs spectral distributions and from the initial amount of the entanglement. For intermediate values of, $x$ the situation is more complicated, and the validity of the secular approximation strongly depends on the expression of the spectral distribution and on the initial amount of entanglement.

The second aim of the paper was the investigation of the entanglement dynamics as a function of the reservoir spectrum, the temperature and the initial amount of entanglement. Essentially we observed the presence of three different behaviors: sudden death of entanglement (ESD), nonMarkovian entanglement revivals and oscillations (NMRev) and oscillations or revivals related only to the secular coefficients (NSRev).

At high-temperatures ESD appears for each value of the initial entanglement. Moreover, for $x \gg 1$ there are no revivals while they are typical of the dynamics for $x \ll 1$. Because in this limit the secular and exact dynamics almost coincide, these revivals are due to the negativity of the master equation coefficients (NMRev). For intermediate values of $x$, the time evolution strongly depends on the initial amount of entanglement. If the entanglement is small $(r<1)$ only ESD is observed. For larger value of $r$, in general, the behavior is characterized by oscillations and revivals. Some of these revivals occur in correspondence of positive value of the timedependent coefficients. Therefore they exist as a consequence of the secular coefficients only (NSRev).

The situation for $T=0$ is characterized by a slower rate of entanglement deterioration. Therefore, for short times, entanglement sudden death and revivals can be observed only for very small initial entanglement $(r \ll 1)$. In these cases, and when $x \gg 1$, ESD exists independently from the reservoir spectrum while for $x \ll 1$ EDS is present in the super-Ohmic case only. Hence, the asymptotic long time Markovian dynamics of entanglement, and therefore also the Markovian prediction about the disentanglement time, may be strongly affected by the non-Markovian short time correlations. When this happens, the non-Markovian theory predicts a finite disentanglement time in contrast to the Markovian prediction. When $r>0.1$ the short time non-Markovian dynamics is characterized by oscillations only, the EOF remaining positive. Therefore, for long times the Markovian prediction of an infinite disentanglement time is recovered.

In recent years, there have been a lot of interest in the entanglement dynamics in $\mathrm{CV}$ quantum channels, both for common and independent reservoirs. Our work finds its place in this context as an attempt to investigate the nonMarkovian short time dynamics of entanglement in different physical scenarios. We believe that our results, showing the effects of different reservoirs on the time evolution of entanglement in $\mathrm{CV}$ quantum channels, will pave the way to 
the implementation of engineered reservoir control schemes as the one recently reported in [41] for qubits.

\section{ACKNOWLEDGMENTS}

S.M. and R.V. acknowledge financial support from the Academy of Finland (Projects No. 115682 and No. 8125004), the Väisälä Foundation, the Magnus Ehrnrooth Foundation, the Emil Aaltonen Foundation and the Turku Collegium of Science and Medicine. This work has been partially supported by the CNR-CNISM convention.

\section{APPENDIX: TIME DEPENDENT COEFFICIENTS AT THE SECOND ORDER IN $\alpha$}

Here we provide the exact analytic expressions of the time-dependent coefficients of the master equation given in Eq. (3). In each subsection, we consider a single reservoir spectral function and evaluate the temperature independent damping coefficient $\gamma(t)$, the diffusion coefficients in the high temperature regime $\Delta_{T}(t)$ and $\Pi_{T}(t)$, and the diffusion coefficients at $T=0, \Delta_{0}(t)$ and $\Pi_{0}(t)$. The expression for $\Gamma(t)$, $\Delta_{\Gamma}(t)$, and the secular terms (20) follow through. We made use of the following special mathematical functions [42]:

$$
\begin{gathered}
\operatorname{Ei}(z)=-\int_{-z}^{+\infty} \frac{e^{-t}}{t} d t \operatorname{Ci}(z)=-\int_{z}^{+\infty} \frac{\cos t}{t} d t \operatorname{Si}(z)=\int_{0}^{z} \frac{\sin t}{t} d t \\
\operatorname{Sih}(z)=\int_{0}^{z} \frac{\sinh t}{t} d t \operatorname{erf}(z)=\frac{2}{\sqrt{\pi}} \int_{0}^{z} e^{-t^{2}} d t
\end{gathered}
$$

\section{Ohmic Reservoir $s=1$}

$$
\begin{aligned}
\gamma(t) & =\frac{\omega_{0} \alpha^{2}}{4}\left\{i e^{-1 / x}\left[\operatorname{Ei}\left(\frac{1-i \tau}{x}\right)-\operatorname{Ei}\left(\frac{1+i \tau}{x}\right)\right]+e^{1 / x}[2 \pi\right. \\
& \left.\left.+i \operatorname{Ei}\left(\frac{i \tau-1}{x}\right)-i \operatorname{Ei}\left(-\frac{1+i \tau}{x}\right)\right]-\frac{4 x \sin (\tau / x)}{1+\tau^{2}}\right\},
\end{aligned}
$$

$$
\begin{aligned}
\Delta_{T}(t)= & -\frac{k_{B} T \alpha^{2}}{\hbar}\left\{i \cosh \left(\frac{1}{x}\right)\left[\operatorname{Ci}\left(\frac{\tau-i}{x}\right)-\operatorname{Ci}\left(\frac{\tau+i}{x}\right)-i \pi\right]\right. \\
& \left.+\sinh \left(\frac{1}{x}\right)\left[\operatorname{Si}\left(\frac{\tau-i}{x}\right)+\operatorname{Si}\left(\frac{\tau+i}{x}\right)\right]\right\}, \\
\Pi_{T}(t)= & \frac{k_{B} T \alpha^{2}}{\hbar}\left\{\operatorname { s i n h } ( \frac { 1 } { x } ) \left[\operatorname{Ci}\left(\frac{\tau-i}{x}\right)+\operatorname{Ci}\left(\frac{\tau+i}{x}\right)-\operatorname{Ci}\left(-\frac{i}{x}\right)\right.\right. \\
& \left.-\operatorname{Ci}\left(\frac{i}{x}\right)\right]+\cosh \left(\frac{1}{x}\right) \times\left[2 \operatorname{Sih}\left(\frac{1}{x}\right)-i \operatorname{Si}\left(\frac{\tau-i}{x}\right)\right. \\
& \left.\left.+i \operatorname{Si}\left(\frac{\tau+i}{x}\right)\right]\right\},
\end{aligned}
$$

$$
\begin{aligned}
\Delta_{0}(t)= & \frac{\omega_{0} \alpha^{2}}{4}\left\{i e^{-1 / x}\left[\operatorname{Ei}\left(\frac{1-i \tau}{x}\right)-\operatorname{Ei}\left(\frac{1+i \tau}{x}\right)\right]-e^{1 / x}[2 \pi\right. \\
& \left.\left.+i \operatorname{Ei}\left(\frac{i \tau-1}{x}\right)-i \operatorname{Ei}\left(-\frac{1+i \tau}{x}\right)\right]+\frac{4 x \tau \cos (\tau / x)}{1+\tau^{2}}\right\},
\end{aligned}
$$

$$
\begin{aligned}
\Pi_{0}(t)= & \frac{\omega_{0} \alpha^{2}}{4}\left\{-e^{-1 / x}\left[\operatorname{Ei}\left(\frac{1-i \tau}{x}\right)+\operatorname{Ei}\left(\frac{1+i \tau}{x}\right)-2 \operatorname{Ei}\left(\frac{1}{x}\right)\right]\right. \\
& +e^{1 / x}\left[2 \operatorname{Ei}\left(-\frac{1}{x}\right)-\operatorname{Ei}\left(\frac{i \tau-1}{x}\right)-\operatorname{Ei}\left(-\frac{1+i \tau}{x}\right)\right] \\
& \left.+\frac{4 x \tau \sin (\tau / x)}{1+\tau^{2}}\right\} .
\end{aligned}
$$

\section{Sub-Ohmic Reservoir $s=1 / 2$}

$$
\begin{aligned}
\gamma(t) & =\frac{\alpha^{2} \omega_{0} \sqrt{\pi}}{4}\left\{\frac{2 i x \sin (t / x)\left(1+i t+\sqrt{1+t^{2}}\right)}{\sqrt{1-i t}(t-i)}\right. \\
& +e^{-1 / x} \sqrt{\pi x}\left[\operatorname{erf}\left((-1)^{3 / 4} \sqrt{\frac{-i+t}{x}}\right)\right. \\
& -i e^{2 / x} \operatorname{erf}\left((-1)^{3 / 4} \sqrt{\frac{-i+t}{x}}\right)+i e^{2 / x} \\
& \left.\left.\times \operatorname{erf}\left((-1)^{3 / 4} \sqrt{\frac{i+t}{x}}\right)+\operatorname{erf}\left((-1)^{1 / 4} \sqrt{\frac{i+t}{x}}\right)\right]\right\},
\end{aligned}
$$

$$
\begin{aligned}
\Delta_{T}(t)= & -\frac{\alpha^{2} \pi k_{B} T}{2 \hbar} \sqrt{x} e^{-1 / x}\left\{\operatorname{erf}\left((-1)^{1 / 4} \sqrt{\frac{i-t}{x}}\right)\right. \\
& -\operatorname{erf}\left((-1)^{1 / 4} \sqrt{\frac{i+t}{x}}\right)+i e^{2 / x} \\
& \left.\times\left[\operatorname{erf}\left((-1)^{3 / 4} \sqrt{\frac{i+t}{x}}\right)-\operatorname{erf}\left((-1)^{3 / 4} \sqrt{\frac{i-t}{x}}\right)\right]\right\}, \\
\Pi_{T}(t)= & \frac{\alpha^{2} \pi k_{B} T}{2 \hbar} \sqrt{x} e^{-1 / x}\left\{e ^ { 2 / x } \left[\operatorname{erf}\left((-1)^{3 / 4} \sqrt{\frac{i-t}{x}}\right)\right.\right. \\
& \left.-\operatorname{erf}\left((-1)^{3 / 4} \sqrt{\frac{i+t}{x}}\right)-2 \operatorname{erf}\left(\sqrt{\frac{1}{x}}\right)\right] \\
& +i\left[\operatorname{erf}\left((-1)^{1 / 4} \sqrt{\frac{i-t}{x}}\right)+\operatorname{erf}\left((-1)^{1 / 4} \sqrt{\frac{i+t}{x}}\right)\right. \\
& \left.\left.-2 \operatorname{erf}\left(i \sqrt{\frac{1}{x}}\right)\right]\right\},
\end{aligned}
$$




$$
\begin{aligned}
\Delta_{0}(t)= & \frac{\alpha^{2} \omega_{0} \sqrt{\pi}}{4}\left\{\frac{2 i x \cos (t / x)}{\sqrt{1+t^{2}}}(\sqrt{1-i t}+\sqrt{1+i t})+e^{-1 / x} \sqrt{x \pi}\right. \\
& \times\left[-\operatorname{erf}\left((-1)^{3 / 4} \sqrt{\frac{-i+t}{x}}\right)+\operatorname{erf}\left((-1)^{1 / 4} \sqrt{\frac{i+t}{x}}\right)\right. \\
& +i e^{2 / x} \operatorname{erf}\left((-1)^{1 / 4} \sqrt{\frac{-i+t}{x}}\right)+i e^{2 / x} \\
& \left.\left.\times \operatorname{erf}\left((-1)^{3 / 4} \sqrt{\frac{i+t}{x}}\right)\right]\right\}, \\
\Pi_{0}(t)= & \frac{\alpha^{2} \omega_{0} \sqrt{\pi}}{4}\left\{-\frac{2 i x \sin (t / x)}{\sqrt{1+t^{2}}}(\sqrt{1-i t}+\sqrt{1+i t})\right. \\
& +e^{1 / x} \sqrt{\pi x}\left[2 e^{2 / x} \operatorname{erf}\left(\sqrt{\frac{1}{x}}\right)\right. \\
& -e^{2 / x} \operatorname{erf}\left((-1)^{1 / 4} \sqrt{\frac{-i+t}{x}}\right)+e^{2 / x} \\
& \times \operatorname{erf}\left((-1)^{3 / 4} \sqrt{\frac{i+t}{x}}\right)+i \operatorname{Erf}\left((-1)^{3 / 4} \sqrt{\frac{-i+t}{x}}\right) \\
& \left.\left.+i e r f\left((-1)^{1 / 4} \sqrt{\frac{i+t}{x}}\right)-2 i e r f\left(i \sqrt{\frac{1}{x}}\right)\right]\right\} \quad(\mathrm{A} 10)
\end{aligned}
$$

\section{Super-Ohmic Reservoir $s=3$}

$$
\begin{aligned}
\gamma(t) & =\frac{\alpha^{2} \omega_{0}}{4 x^{2}\left(1+t^{2}\right)^{3}}\left\{8 x^{2}\left(1+t^{2}\right) t \cos \left(\frac{t}{x}\right)+4 x\left[-\left(1+t^{2}\right)^{2}\right.\right. \\
& \left.+2\left(3 t^{2}-1\right) x^{2}\right] \sin \left(\frac{t}{x}\right)+e^{-1 / x}\left(1+t^{2}\right)^{3}\left[2 e^{2 / x} \pi\right. \\
& +i \operatorname{Ei}\left(\frac{1-i t}{x}\right)-i e^{2 / x} \operatorname{Ei}\left(-\frac{1+i t}{x}\right)-i \operatorname{Ei}\left(\frac{1+i t}{x}\right) \\
& \left.\left.+i e^{2 / x} \operatorname{Ei}\left(-\frac{1-i t}{x}\right)\right]\right\},
\end{aligned}
$$

$$
\begin{aligned}
& \Delta_{T}(t)=\frac{\alpha^{2} k_{B} T}{2 \hbar x^{2}\left(1+t^{2}\right)^{2}}\left\{8 x^{2} t \cos \left(\frac{t}{x}\right)-4\left(1+t^{2}\right) x \sin \left(\frac{t}{x}\right)\right. \\
& +e^{-1 / x}\left(1+t^{2}\right)^{2}\left[2 e^{2 / x} \pi+i \operatorname{Ei}\left(\frac{1-i t}{x}\right)-i e^{2 / x}\right. \\
& \left.\left.\times \operatorname{Ei}\left(-\frac{1+i t}{x}\right)-i \operatorname{Ei}\left(\frac{1+i t}{x}\right)+i e^{2 / x} \operatorname{Ei}\left(-\frac{1-i t}{x}\right)\right]\right\}, \\
& \Pi_{T}(t)=\frac{\alpha^{2} k_{B} T}{2 \hbar x^{2}\left(1+t^{2}\right)^{2}}\left\{4 x\left(1+t^{2}\right) \cos \left(\frac{t}{x}\right)+8 t x^{2} \sin \left(\frac{t}{x}\right)\right. \\
& -e^{-1 / x}\left(1+t^{2}\right)^{2}\left[4 e^{1 / x} x+2 e^{2 / x} \operatorname{Ei}\left(-\frac{1}{x}\right)-2 \operatorname{Ei}\left(\frac{1}{x}\right)\right. \\
& +\operatorname{Ei}\left(\frac{1-i t}{x}\right)-e^{2 / x} \operatorname{Ei}\left(-\frac{1+i t}{x}\right)+\operatorname{Ei}\left(\frac{1+i t}{x}\right) \\
& \left.\left.-e^{2 / x} \operatorname{Ei}\left(-\frac{1-i t}{x}\right)\right]\right\} \text {, } \\
& \Delta_{0}(t)=\frac{\alpha^{2} \omega_{0}}{2 x^{2}}\left\{\frac { 2 x } { ( 1 + t ^ { 2 } ) ^ { 3 } } \left[-\left(1-t^{4}\right) x \sin \left(\frac{t}{x}\right)+t \cos \left(\frac{t}{x}\right)\right.\right. \\
& \left.\times\left(1+2 t^{2}+t^{4}+6 x^{2}-2 x^{2} t^{2}\right)\right]+i \sinh \left(\frac{1}{x}\right) \\
& \times\left[\mathrm{Ci}\left(\frac{-i+t}{x}\right)-\mathrm{Ci}\left(\frac{i+t}{x}\right)+i \pi\right]+\cosh \left(\frac{1}{x}\right) \\
& \left.\times\left[\operatorname{Si}\left(\frac{-i+t}{x}\right)+\operatorname{Si}\left(\frac{i+t}{x}\right)\right]\right\}, \\
& \Pi_{0}(t)=\frac{\alpha^{2} \omega_{0}}{2 x^{2}}\left\{-2 x^{2}+\frac{2 x}{\left(1+t^{2}\right)^{3}}\left[\left(1-t^{4}\right) x \cos \left(\frac{t}{x}\right)+t \sin \left(\frac{t}{x}\right)\right.\right. \\
& \left.\times\left(1+2 t^{2}+t^{4}+6 x^{2}-2 x^{2} t^{2}\right)\right]-\cosh \left(\frac{1}{x}\right) \\
& \times\left[\mathrm{Ci}\left(\frac{-i+t}{x}\right)+\mathrm{Ci}\left(\frac{i+t}{x}\right)-i \pi\right]+\sinh \left(\frac{1}{x}\right) \\
& \times\left[-2 \operatorname{Sin}\left(\frac{1}{x}\right)+i \operatorname{Si}\left(\frac{-i+t}{x}\right)\right. \\
& \left.\left.-i \operatorname{Si}\left(\frac{i+t}{x}\right)\right]\right\} \text {. }
\end{aligned}
$$

[1] M. A. Nielsen and I. L. Chuang, Quantum Computation and Quantum Information (Cambridge University Press, Cambridge, 2000).

[2] H.-P. Breuer and F. Petruccione, The Theory of Open Quantum Systems (Oxford University Press, Oxford, 2002).

[3] U. Weiss, Quantum Dissipative Systems (World Scientific, Singapore, 1999).

[4] W. H. Zurek, Rev. Mod. Phys. 75, 715 (2003).

[5] Q. A. Turchette, C. J. Myatt, B. E. King, C. A. Sackett, D.
Kielpinski, W. M. Itano, C. Monroe, and D. J. Wineland, Phys. Rev. A 62, 053807 (2000); C. J. Myatt et al., Nature (London) 403, 269 (2000).

[6] S. Diehl, A. Micheli, A. Kantian, B. Kraus, H.-P. Büchler, and P. Zoller, Nat. Phys. 4, 878 (2008).

[7] S. L. Braunstein and P. van Loock, Rev. Mod. Phys. 77, 513 (2005).

[8] J. S. Prauzner-Bechcicki, J. Phys. A 37, L173 (2004).

[9] S.-H. Xiang, B. Shao, and K.-H. Song, Phys. Rev. A 78, 
052313 (2008).

[10] A. Serafini, F. Illuminati, M. G. A. Paris, and S. De Siena, Phys. Rev. A 69, 022318 (2004); A. Serafini, M. G. A. Paris, F. Illuminati, and S. De Siena, J. Opt. B: Quantum Semiclassical Opt. 7, R19 (2005).

[11] P. J. Dodd and J. J. Halliwell, Phys. Rev. A 69, 052105 (2004).

[12] P. J. Dodd, Phys. Rev. A 69, 052106 (2004).

[13] T. Hiroshima, Phys. Rev. A 63, 022305 (2001).

[14] J. P. Paz and A. J. Roncaglia, Phys. Rev. Lett. 100, 220401 (2008).

[15] J. P. Paz and A. J. Roncaglia, Phys. Rev. A 79, 032102 (2009).

[16] K. Shiokawa, Phys. Rev. A 79, 012308 (2009).

[17] J.-H. An and W.-M. Zhang, Phys. Rev. A 76, 042127 (2007).

[18] M. Ban, J. Phys. A 39, 1927 (2006); Phys. Lett. A 359, 402 (2006).

[19] K.-L. Liu and H.-S. Goan, Phys. Rev. A 76, 022312 (2007).

[20] J.-H. An, Y. Yeo, W.-M. Zhang, and C. H. Oh, J. Phys. A: Math. Theor. 42, 015302 (2009).

[21] S. Maniscalco, S. Olivares, and M. G. A. Paris, Phys. Rev. A 75, 062119 (2007).

[22] C. H. Bennett, D. P. DiVincenzo, J. A. Smolin, and W. K. Wootters, Phys. Rev. A 54, 3824 (1996).

[23] G. Giedke, M. M. Wolf, O. Kruger, R. F. Werner, and J. I. Cirac, Phys. Rev. Lett. 91, 107901 (2003).

[24] P. Marian and T. A. Marian, Phys. Rev. Lett. 101, 220403 (2008).

[25] B. L. Hu, J. P. Paz, and Y. Zhang, Phys. Rev. D 45, 2843 (1992).

[26] F. Intravaia, S. Maniscalco, and A. Messina, Phys. Rev. A 67, 042108 (2003).

[27] R. P. Feynman and F. L. Vernon, Ann. Phys. (N.Y.) 24, 118
(1963).

[28] J. Paavola, J. Piilo, K.-A. Suominen, and S. Maniscalco, Phys. Rev. A 79, 052120 (2009).

[29] S. Maniscalco, J. Piilo, and K.-A. Suominen, Eur. Phys. J. D 55, 181 (2009).

[30] R. Alicki, M. Horodecki, P. Horodecki, and R. Horodecki, Phys. Rev. A 65, 062101 (2002).

[31] T. Hu and J. H. Eberly, Science 323, 598 (2009).

[32] B. Bellomo, R. Lo Franco, and G. Compagno, Phys. Rev. Lett. 99, 160502 (2007).

[33] L. Mazzola, S. Maniscalco, J. Piilo, K.-A. Suominen, and B. M. Garraway, Phys. Rev. A 79, 042302 (2009).

[34] S. Maniscalco, F. Francica, R. L. Zaffino, N. Lo Gullo, and F. Plastina, Phys. Rev. Lett. 100, 090503 (2008).

[35] F. Francica, S. Maniscalco, J. Piilo, F. Plastina, and K.-A. Suominen, Phys. Rev. A 79, 032310 (2009).

[36] K. Härkönen, F. Plastina, and S. Maniscalco, Phys. Rev. A 80, 033841 (2009).

[37] J. Piilo, S. Maniscalco, K. Härkönen, and K.-A. Suominen, Phys. Rev. Lett. 100, 180402 (2008).

[38] J. Piilo, K. Härkönen, S. Maniscalco, and K.-A. Suominen, Phys. Rev. A 79, 062112 (2009).

[39] S. Maniscalco, J. Piilo, F. Intravaia, F. Petruccione, and A. Messina, Phys. Rev. A 70, 032113 (2004).

[40] J. Jing, Z.-G. Lu, and Z. Ficek, Phys. Rev. A 79, 044305 (2009).

[41] M. J. Biercuk, H. Uys, A. P. VanDevender, N. Shiga, W. M. Itano, and J. J. Bollinger, Nature (London) 458, 996 (2009).

[42] M. Abramowitz, Hanbook of Mathematical Functions, edited by I. A. Stegun (Dover Publication, New York, 1965). 